\title{
On molecular topological descriptors of certain families of nanostar dendrimers
}

\author{
Muhammad Imran ${ }^{\mathrm{a}, *}$ \\ |Syed Ahtsham Ul Haq Bokharyb \\ |Sadia Manzoor ${ }^{\mathrm{c}} \mid$ Muhammad Kamran \\ Siddiquid $^{\mathrm{P}}$
}

aDepartment of Mathematical Sciences, United Arab Emirates University P.O. BOX 15551, Al Ain, United Arab Emirates

${ }^{b}$ Centre for Advanced Studies in Pure and Applied Mathematics, Bahauddin Zakariya University, Multan, Pakistan

'Centre for Advanced Studies in Pure and Applied Mathematics, Bahauddin Zakariya University, Multan, Pakistan

dDepartment of Mathematics, Comsats Institute of Information Technology, Sahiwal, Pakistan

*Corresponding Author:

Muhammad Imran

Email:imrandhab@gmail.com

Tel.: +923334736997

In this article, we study the degree-based molecular topological indices for some infinite families of nanostar dendrimers. We derive the analytical closed formulae for these classes of complex chemical networks. These results are very helpful in understanding and predicting the physico-chemical properties for these chemical structures (Nanostar dendrimers $N S_{2}[n]$, $\left.N S_{3}[n], D_{2}[n]\right]$.

\section{Introduction}

Graph theory has provided the chemist with a variety of useful tools, such as topological indices and topological polynomials. Molecules and molecular compounds are often modeled by a molecular graph. Cheminformatics is new subject which is a combination of chemistry, mathematics and information science. It studies quantitative structure-activity (QSAR) and structureproperty (QSPR) relationships that are used to predict the biological activities and properties of different chemical compounds. The application of molecular structure descriptors is nowadays a standard procedure in the study of structure-property relations, especially in QSPR/QSAR study. In the last few years, the number of proposed molecular descriptors is rapidly growing due to the chemical significance of these descriptors. These descriptors correlate certain chemical and physical properties of chemical compounds. A good model for the stability of linear and branched alkanes as well as the strain energy of cycloalkanes is provided by the atom-bond connectivity $(A B C)$ index. For certain physico-chemical properties like boiling point, entropy, enthalpy of vaporization, standard enthalpy of vaporization, enthalpy of formation and acentric factor, the predictive power of geometric-arithmetic $(G A)$ index is better than predictive power of other connectivity indices. Topological characterization of chemical structures allows the classification of molecules and modelling unknown structures with desired properties. Molecules and molecular compounds are often modeled by molecular graphs.

A molecular graph is a representation of the structural formula of a chemical compound in terms of graph theory, whose vertices correspond to the atoms of the compound and edges correspond to chemical bonds. The combination of chemistry, mathematics and information science which studies QSAR/QSPR relationships is known as cheminformatics. Many chemical and physical properties of the chemical compounds can be 
found with the help of QSAR /QSPR models. The topological indices such as Wiener, atombond connectivity $(A B C)$, and geometricarithmetic (GA) indices are used to correlate different chemical and physical properties like the boiling point, molecular weight, vapour pressure, $\pi$-electrom energy etc.

Nanobiotechnology is a rapidly advancing area of scientific and technological opportunity that applies the tools and processes of nanofabrication to build devices for studying biosystems. Dendrimers are one of the main objects of this new area of science. A dendrimer is an artificially manufactured or synthesized molecule built up from branched units called monomers using a nanoscale fabrication process. Dendrimers are recognized as one of the major commercially available nanoscale building blocks, large and complex molecules with very well-defined chemical structure. From a polymer chemistry point of view, dendrimers are nearly perfect monodisperse macromolecules with a regular and highly branched three-dimensional architecture. They consist of three major architectural components, core, branches and end groups. New branches emitting from a central core are added in steps until a tree-like structure is created. The nanostar dendrimer is a part of a new group of macroparticles that appear to be photon funnels just like artificial antennas. These macromolecules and more precisely those containing phosphorus are used in the formation of nanotubes, micro and macrocapsules, nanolatex, coloured glasses, chemical sensors, modified electrodes and so on.

\section{Materials and methods}

A graph can be recognized by a numeric number, a polynomial, a sequence of numbers or a matrix. A topological index is a numeric quantity associated with a graph which characterizes the topology of graph and is invariant under graph automorphism. There are some major classes of topological indices such as distance-based topological indices, degree-based topological indices and counting related polynomials and indices of graphs. Among these classes, degree-based topological indices are of great importance and play a vital role in chemical graph theory and particularly in chemistry. In more precise way, a topological index $\operatorname{Top}(G)$ of a graph, is a number with the property that for every graph $\mathrm{H}$ isomorphic to $G$, we have $\operatorname{Top}(H)=\operatorname{Top}(G)$. The concept of topological indices came from Wiener (1947) who named this index as path number [28] while he was working on boiling point of paraffin. Later on, the path number was renamed as Wiener index and the theory of topological indices started [6-8, 25, and 26].

In this article, $G$ is considered to be network with vertex set $V(G)$ and edge set $E(G), d(u)$ is the degree of vertex $u \in V(G)$ and

$$
S_{u}=\sum_{v \in N_{G}(u)} d(v)
$$

where $N_{G}(u)=\{v \in V(G) \mid u v \in E(G)\}$. The notations used in this article are mainly taken from books of Diudea et al. (2001) [11].

Let $G$ be a graph. Then the Wiener index of $G$ is defined as [28]:

$$
W(G)=\frac{1}{2} \sum_{(u, v)} d(u, v)
$$

where $(u, v)$ is any ordered pair of vertices in $G$ and $d(u, v)$ is $u-v$ geodesic.

One of the well-known degree-based topological indices is atom-bond connectivity $(A B C)$ index introduced by Estrada et al. (1998) and defined as [12]:

$$
A B C(G)=\sum_{u v E(G)} \sqrt{\frac{d(u)+d(v)-2}{d(u) d(v)}}
$$

Another well-known connectivity topological descriptor is geometric-arithmetic (GA) index which was introduced by Vukičević et al., 2009 , and defined as $[13,27]$ : 


$$
G A(G)=\sum_{u v \in E(G)} \frac{2 \sqrt{d(u) d(v)}}{d(u)+d(v)}
$$

$A B C_{4}$ and $G A_{5}$ indices can be computed if we are able to find the edge partition of these interconnection chemical networks based on sum of the degrees of end vertices of each edge in these graphs. The fourth version of $A B C$ index is introduced by Ghorbani et al., 2010, and defined as [14]:

$$
A B C_{4}(G)=\sum_{u v \in E(G)} \sqrt{\frac{S_{u}+S_{v}-2}{S_{u} S_{v}}}
$$

Recently, fifth version of $G A$ index is proposed by Graovac et al., 2011, and defined as [15]:

$$
G A_{5}(G)=\sum_{u v \in E(G)} \frac{2 \sqrt{S_{u} S_{v}}}{S_{u}+S_{v}}
$$

Imran et al., 2014, studied various degreebased topological indices for various networks like silicates, hexagonal, honeycomb and oxide. Nowadays, there is an extensive research activity on $A B C$ and $G A$ indices and their variants. For further study of topological indices of various graphs and chemical structures, see [2-5, 16-24].

In this paper, we study the certain degreebased molecular topological indices for complex chemical networks like some infinite families of nanostar dendrimers. We derive the analytical closed formulae for these classes of complex chemical networks.

\section{Results and discussion}

The topological descriptors of nanostar dendrimers $N S_{2}[n]$

In this section, we first calculate the $A B C_{4}$ and $G A_{5}$ indices of nanostar dendrimers denoted by $N S_{2}[n]$. The number of vertices and edges in $N S_{2}[n]$ are $16 \times 2^{n_{-}}-4$ and $18 \times 2^{n-5}[1,9]$. Denote an edge connecting a vertex $\mathrm{i}$ to a vertex $\mathrm{j}$ by $(i, j)$-edge. Where $\mathrm{n}_{\mathrm{i}}$ denote the vertex i and $s_{i j}$ are the number of $(i, j)$-edges. The nanostar $\mathrm{NS}_{2}[3]$ is shown in the Figure 1.

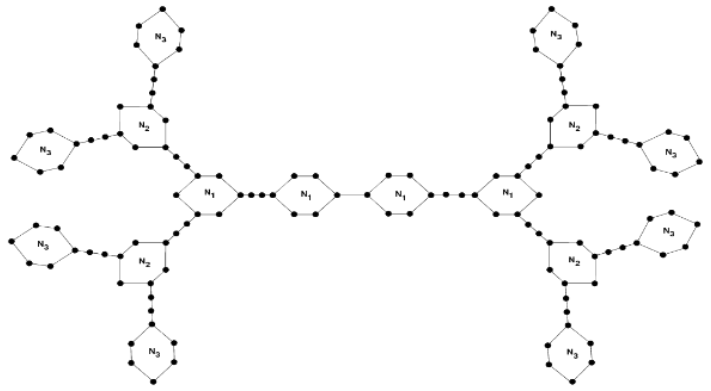

FIGURE 1 The nanostar $\mathrm{NS}_{2}[3]$

TABLE 1 Edge partition of nanostar $N S_{2}[n]$ based on degree sum of neighbors of end vertices of each edge

\begin{tabular}{cc}
$\left(\boldsymbol{S}_{\boldsymbol{u}}, \boldsymbol{S}_{\boldsymbol{v}}\right)$ where $\boldsymbol{u v} \boldsymbol{E} \boldsymbol{E}(\boldsymbol{G})$ & Number of edges \\
$(4,4)$ & $2 \times 2^{n}$ \\
$(4,5)$ & $2 \times 2^{n}$ \\
$(5,5)$ & $2 \times 2^{n}+2$ \\
$(5,6)$ & $6 \times 2^{n}$ \\
$(7,7)$ & 1 \\
$(5,7)$ & 4 \\
$(6,6)$ & $6 \times 2^{n-12}$ \\
\hline
\end{tabular}

Theorem 1 Let $n \in \mathrm{N}$, then $A B C_{4}$ index for Nanostar $N S_{2}[n]$ is given by

$$
\begin{aligned}
& A B C_{4}\left(N S_{2}[n]\right)=\left(\frac{\sqrt{2}}{5}+\frac{3}{\sqrt{30}}\right) 2^{n+2} \\
& +\left(\frac{6}{\sqrt{10}}+\sqrt{10}+\frac{\sqrt{6}}{2}+\sqrt{\frac{7}{5}}\right) 2^{n}+4\left(\frac{\sqrt{2}}{5}+\sqrt{\frac{10}{35}}-\frac{\sqrt{10}}{2}\right)
\end{aligned}
$$

Proof. The graph $\mathrm{NS}_{2}[\mathrm{n}]$ have edge partition of the form $(4,4),(4,5),(5,5),(6,5),(5,7)$, $(6,7),(6,6)$. We apply the information given in Table 1 to compute the $A B C_{4}$ index of $\mathrm{NS}_{2}[n]$. Since we have

$$
A B C_{4}\left(N S_{2}[n]\right)=\sum_{u v \in[G)} \sqrt{\frac{S_{u}+S_{v}-2}{S_{u} S_{v}}} .
$$

This implies that

$$
\begin{aligned}
& A B C_{4}\left(N S_{2}[n]\right)=\left(2^{n+1}+2\right) \sqrt{\frac{5+5-2}{5 \times 5}} \\
& +2\left(2^{n+1}+2\right) \sqrt{\frac{5+6-2}{5 \times 6}}+\left(6 \times 2^{n}-12\right) \sqrt{\frac{6+6-2}{6 \times 6}}+ \\
& \left(2 \times 2^{n}\right) \sqrt{\frac{4+4-2}{4 \times 4}}+\left(2 \times 2^{n}\right) \sqrt{\frac{5+4-2}{5 \times 4}} \\
& +1 \times \sqrt{\frac{7+7-2}{7 \times 7}}+4 \times \sqrt{\frac{5+7-2}{5 \times 7}} .
\end{aligned}
$$


Which can be reduced to the following after simplification.

$$
\begin{aligned}
& A B C_{4}\left(N S_{2}[n]\right)=\left(\frac{\sqrt{2}}{5}+\frac{3}{\sqrt{30}}\right) 2^{n+2} \\
& +\left(\frac{6}{\sqrt{10}}+\sqrt{10}+\frac{\sqrt{6}}{2}+\sqrt{\frac{7}{5}}\right) 2^{n}+4\left(\frac{\sqrt{2}}{5}+\sqrt{\frac{10}{35}}-\frac{\sqrt{10}}{2}\right) .
\end{aligned}
$$

Theorem 2 Consider the nanostar $N S_{2}[n]$, then its $G A_{5}$ index is computed as

$$
\begin{aligned}
& G A_{5}\left(N S_{2}[n]\right)=\left(2+\frac{8 \sqrt{5}}{9}+\frac{4 \sqrt{30}}{11}\right) 2^{n} \\
& +\left(1+4 \frac{\sqrt{30}}{11}\right) 2^{n+1}+\left(\frac{2 \sqrt{35}}{3}+1\right)
\end{aligned}
$$

Proof. By using the edge partition given in Table 1 , the $G A_{5}$ index of nanostar graph $N S_{2}[n]$ is calculated as we have

$$
\begin{aligned}
& G A_{5}\left(N S_{2}[n]\right)=\frac{2 \sqrt{4 \times 4}}{4+4}\left(2 \times 2^{n}\right)+\frac{2 \sqrt{5 \times 4}}{5+4}\left(2 \times 2^{n}\right) \\
& +\frac{2 \sqrt{5 \times 5}}{5+5}\left(2^{n+1}+2\right)+\frac{2 \sqrt{5 \times 6}}{5+6} 2 \times\left(2^{n+1}+2^{n}\right)+ \\
& \frac{2 \sqrt{7 \times 7}}{7+7} \times 1+\frac{2 \sqrt{5 \times 7}}{5+7} \times 4+\frac{2 \sqrt{6 \times 6}}{6+6}\left(6 \times 2^{n}-12\right) .
\end{aligned}
$$

After simplification, we get

$$
\begin{aligned}
& G A_{5}\left(N S_{2}[n]\right)=\left(2+\frac{8 \sqrt{5}}{9}+\frac{4 \sqrt{30}}{11}\right) 2^{n} \\
& +\left(1+4 \frac{\sqrt{30}}{11}\right) 2^{n+1}+\left(\frac{2 \sqrt{35}}{3}+1\right) .
\end{aligned}
$$

The topological descriptors of nanostar dendrimers $\mathrm{NS}_{3}[\mathrm{n}]$

Now, we consider another type of nanostar $N S_{3}[n]$, where $\mathrm{n} \geq 1$. The graph of $N S_{3}[n]$ contains $18 \times 2^{n-12}$ vertices and $21 \times 2^{n-15}$ edges. The graph of nanostar $N S_{3}[n]$ is shown in the Figure 2.

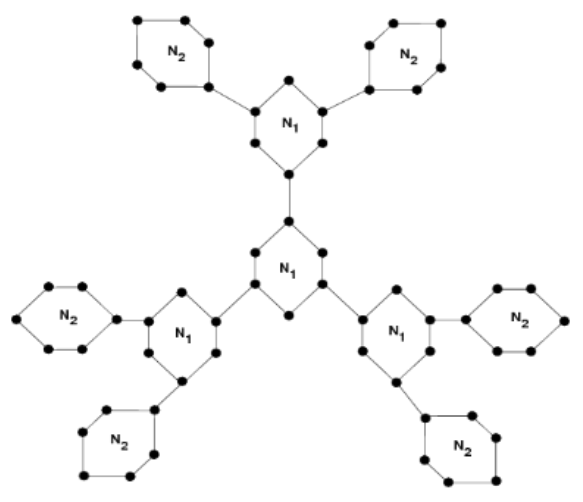

FIGURE 2 The Nanostar $N S_{3}[2]$
TABLE 2 Edge partition for the graph of nanostar $N S_{3}[n]$ based on degree sum of neighbors of end vertices of each edge

$\left(S_{u}, S_{v}\right)$ where $u v \in E(G) \quad$ Number of Edges
$(4,4)$

$(7,7)$
$3 \times 2^{n}$

$3 \times 2^{n}$

$3 \times 2^{n}$

$9 \times 2^{n-12}$

$3 \times 2^{n-3}$
In the next two theorems, we calculate the $A B C_{4}$ and $G A_{5}$ indices of nanostar $N S_{3}[n]$.

Theorem 3 For $\mathrm{n} \geq 1$, the $A B C_{4}$ index of nanostar $\mathrm{NS}_{3}[\mathrm{n}]$ is

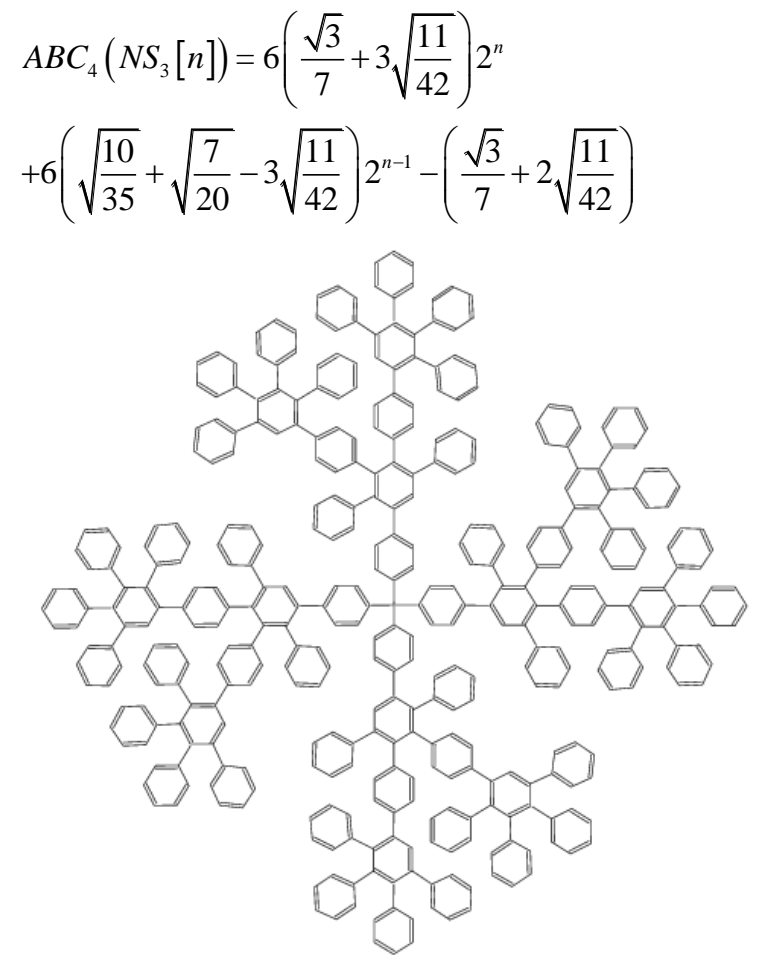

FIGURE 3 The polyphenylene dendrimers $D_{4}[n]$

Proof. For the nanostar $\mathrm{NS}_{3}[\mathrm{n}]$, we have edges of the form $(4,4),(4,5),(5,7),(6,7)$ and $(7,7)$. We use the values given in Table 2 to derive the formula for $A B C_{4}\left(N S_{3}[n]\right)$. We have

$$
\left(9 \times 2^{n}-12\right) \sqrt{\frac{6+7-2}{6 \times 7}}+3\left(2^{n}-1\right) \sqrt{\frac{7+7-2}{7 \times 7}} .
$$

After an easy simplification, we have 
$A B C_{4}\left(N S_{3}[n]\right)=6\left(\frac{\sqrt{3}}{7}+3 \sqrt{\frac{11}{42}}\right) 2^{n}$

$+6\left(\sqrt{\frac{10}{35}}+\sqrt{\frac{7}{20}}-3 \sqrt{\frac{11}{42}}\right) 2^{n-1}-\left(\frac{\sqrt{3}}{7}+2 \sqrt{\frac{11}{42}}\right)$.

Theorem 4 Let $\mathrm{n} \geq 1$, then $G A_{5}$ index of nanostar $N S_{3}[n]$ is calculated as

$$
\begin{aligned}
& G A_{5}\left(N S_{3}[n]\right)=3\left(1+12 \frac{\sqrt{42}}{13}\right) 2^{n} \\
& +\left(36 \frac{\sqrt{42}}{13}+4 \frac{\sqrt{5}}{9}+\sqrt{35}+6\right) 2^{n-1}-36 \sqrt{42} .
\end{aligned}
$$

TABLE 3 Edge partition of the graph $D_{4}[n]$ which depend on the degree sum of vertices having unit distance from each edge

\begin{tabular}{cc}
\hline$\left(\boldsymbol{S}_{u}, \boldsymbol{S}_{v}\right)$ where $u v \in E(G)$ & Number of Edges \\
\hline$(4,4)$ & $24 \times 2^{n-16}$ \\
$(4,5)$ & $24 \times 2^{n-16}$ \\
$(5,5)$ & $8 \times 2^{n-8}$ \\
$(5,7)$ & $40 \times 2^{n-32}$ \\
$(6,8)$ & $8 \times 2^{n-8}$ \\
$(7,7)$ & 2 \\
$(7,8)$ & $8 \times 2^{n-8}$ \\
$(7,9)$ & $12 \times 2^{n-12}$ \\
$(8,9)$ & $8 \times 2^{n-8}$ \\
$(9,9)$ & $8 \times 2^{n-8}$ \\
\hline
\end{tabular}

Proof. By using the edge partition given in Table 2, we calculate the $G A_{5}$ index of $\mathrm{NS}_{3}[\mathrm{n}]$ as follows,

$$
\begin{aligned}
& G A_{5}\left(N S_{3}[n]\right)=\sum_{u v \in E(G)} \frac{2 \sqrt{S_{u} S_{V}}}{S_{u}+S_{v}}=3 \times 2^{n} \times 2 \frac{\sqrt{4 \times 4}}{4+4} \\
& +3 \times 2^{n} \times 2 \frac{\sqrt{4 \times 5}}{4+5}+3 \times 2^{n} \times 2 \frac{\sqrt{5 \times 7}}{5+7}+ \\
& \left(9 \times 2^{n}-12\right) \times 2 \frac{\sqrt{6 \times 7}}{6+7}+3\left(2^{n}-1\right) \times 2 \frac{\sqrt{7 \times 7}}{7+7} .
\end{aligned}
$$

After an easy simplification, we have

$$
\begin{aligned}
& G A_{5}\left(N S_{3}[n]\right)=3\left(1+12 \frac{\sqrt{42}}{13}\right) 2^{n} \\
& +\left(36 \frac{\sqrt{42}}{13}+4 \frac{\sqrt{5}}{9}+\sqrt{35}+6\right) 2^{n-1}-36 \sqrt{42} .
\end{aligned}
$$

The topological descriptors of polyphenylene dendrimers $D_{4}[n]$.

We shall now determine $A B C_{4}$ and $G A_{5}$ indices of polyphenylene dendrimers. In Figure 3, the graph of polyphenylene dendrimers $D_{4}[n]$ of generations $G_{2}$ with 2 growth stages is shown. By using edge partition given in Table 3 , we compute the $A B C_{4}$ and $G A_{5}$ indices of polyphenylene dendrimers.

TABLE 4 Edge partition of the graph $D_{2}[n]$ which depend on the degree sum of vertices having unit distance from each edge

$\left(S_{u}, S_{v}\right)$ where $u v \in E(G) \quad$ Number of Edges

$\begin{array}{cc}(4,4) & 32 \times 2^{n-32} \\ (4,5) & 32 \times 2^{n-32} \\ (5,5) & 16 \times 2^{n-32} \\ (5,7) & 32 \times 2^{n} \\ (6,7) & 12 \\ (6,8) & 8 \times 2^{n-8} \\ (7,7) & 1 \\ (7,8) & 8 \times 2^{n-8} \\ (7,9) & 12 \times 2^{n-12} \\ (8,9) & 8 \times 2^{n-8} \\ (9,9) & 8 \times 2^{n-8}\end{array}$

Theorem 5 Let $\mathrm{n} \geq 1$, then the $A B C_{4}$ index of polyphenylene dendrimers is given by

$$
\begin{gathered}
A B C_{4}\left(D_{4}[n]\right)=2^{n+1}\left(6 \sqrt{\frac{7}{5}}+6 \sqrt{12}+\frac{8 \sqrt{2}}{5}\right. \\
\left.+20 \sqrt{\frac{10}{35}}+2 \sqrt{\frac{13}{14}}+\frac{2}{3} \sqrt{\frac{15}{2}}+\frac{25}{9}\right)- \\
4\left(2 \sqrt{\frac{7}{5}}+\sqrt{12}+\frac{4 \sqrt{2}}{5}+16 \sqrt{\frac{10}{35}}+\sqrt{\frac{13}{14}}+\frac{1}{3} \sqrt{\frac{15}{2}}+\frac{8}{9}\right) .
\end{gathered}
$$

Proof. The graph $D_{4}[n]$ has edges of the form $(4,4),(4,5),(5,7),(6,8),(7,7),(7,8),(8$, 9) and $(9,9)$.

$$
\begin{gathered}
A B C_{4}\left(D_{4}[n]\right)=\sum_{u v E(G)} \sqrt{\frac{S_{u}+S_{v}-2}{S_{u} S_{v}}} \\
=\sqrt{\frac{4+5-2}{4 \times 5}}\left(242^{n}-16\right)+\sqrt{\frac{4+4-2}{4 \times 4}}\left(242^{n}-16\right)+\sqrt{\frac{9+9-2}{9 \times 9}}\left(82^{n}-8\right) \\
+\sqrt{\frac{5+5-2}{5 \times 5}}\left(82^{n}-8\right)+\sqrt{\frac{8+9-2}{8 \times 9}}\left(82^{n}-8\right)+\sqrt{\frac{6+8-2}{6 \times 8}}\left(82^{n}-8\right) \\
+\sqrt{\frac{5+7-2}{5 \times 7}}\left(402^{n}-32\right)+\sqrt{\frac{7+8-2}{7 \times 8}}\left(82^{n}-8\right)+\sqrt{\frac{7+9-2}{7 \times 9}}\left(122^{n}-12\right) .
\end{gathered}
$$

After an easy simplification, we get 


$$
\begin{gathered}
A B C_{4}\left(D_{4}[n]=2^{n+1}\left(6 \sqrt{\frac{7}{5}}+6 \sqrt{12}+\frac{8 \sqrt{2}}{5}\right.\right. \\
\left.+20 \sqrt{\frac{10}{35}}+2 \sqrt{\frac{13}{14}}+\frac{2}{3} \sqrt{\frac{15}{2}}+\frac{25}{9}\right)- \\
4\left(2 \sqrt{\frac{7}{5}}+\sqrt{12}+\frac{4 \sqrt{2}}{5}+16 \sqrt{\frac{10}{35}}+\sqrt{\frac{13}{14}}+\frac{1}{3} \sqrt{\frac{15}{2}}+\frac{8}{9}\right) .
\end{gathered}
$$

Theorem 6 Consider the polyphenylene dendrimers $D_{4}[n]$, then

$$
\begin{aligned}
& G A_{5}\left(D_{4}[n]\right)=\left(\frac{\sqrt{35}}{3}+\frac{32 \sqrt{14}}{15}+\frac{9 \sqrt{7}}{2}\right. \\
& \left.+\frac{96 \sqrt{2}}{17}+\frac{16 \sqrt{3}}{7}+\frac{32 \sqrt{5}}{3}+40\right) 2^{n} \\
& -\left(\frac{64 \sqrt{5}}{9}+\frac{16 \sqrt{35}}{3}+\frac{32 \sqrt{14}}{15}+\frac{9 \sqrt{7}}{2}+\frac{96 \sqrt{2}}{17}+\frac{16 \sqrt{3}}{7}+34\right) \text {. }
\end{aligned}
$$

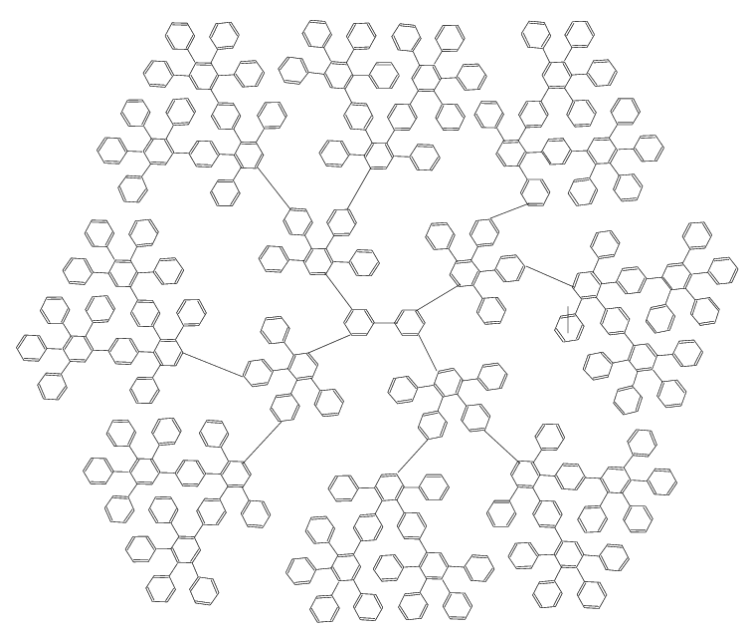

FIGURE 4 The polyphenylene dendrimers $D_{2}[n]$

Proof. Let $\mathrm{n} \geq 1$, then according to the edge partition given in Table 3 , we have

$$
\begin{gathered}
G A_{5}\left(D_{4}[n]\right)=\sum_{u v \in E(G)} \frac{2 \sqrt{S_{u} S_{v}}}{S_{u}+S_{v}} \\
=\frac{2 \sqrt{4 \times 5}}{4+5}\left(242^{n}-16\right)+\frac{2 \sqrt{4 \times 4}}{4+4}\left(242^{n}-16\right)+\frac{2 \sqrt{5 \times 5}}{5+5}\left(82^{n}-8\right) \\
+\frac{2 \sqrt{5 \times 7}}{5+7}\left(402^{n}-32\right)+\frac{2 \sqrt{7 \times 8}}{7+8}\left(82^{n}-8\right) \\
+\frac{2 \sqrt{7 \times 9}}{7+9}\left(122^{n}-12\right)+\frac{2 \sqrt{8 \times 9}}{8+9}\left(82^{n}-8\right) \\
+\frac{2 \sqrt{8 \times 6}}{8+6}\left(82^{n}-8\right)+\frac{2 \sqrt{9 \times 9}}{9+9}\left(82^{n}-8\right)+\frac{2 \sqrt{7 \times 7}}{7+7} \times 2
\end{gathered}
$$

After simplification, we get

$$
\begin{gathered}
G A_{5}\left(D_{4}[n]\right)=\left(\frac{\sqrt{35}}{3}+\frac{32 \sqrt{14}}{15}+\frac{9 \sqrt{7}}{2}\right. \\
\left.+\frac{96 \sqrt{2}}{17}+\frac{16 \sqrt{3}}{7}+\frac{32 \sqrt{5}}{3}+40\right) 2^{n} \\
-\left(\frac{64 \sqrt{5}}{9}+\frac{16 \sqrt{35}}{3}+\frac{32 \sqrt{14}}{15}+\frac{9 \sqrt{7}}{2}+\frac{96 \sqrt{2}}{17}+\frac{16 \sqrt{3}}{7}+34\right) .
\end{gathered}
$$

The topological descriptors of polyphenylene dendrimers $D_{2}[n]$

In this section, we compute the $A B C_{4}$ and $G A_{5}$ indices of another type of polyphenylene dendrimer, denoted by $D_{2}[n]$. Figure 4 shows the graph of polyphenylene dendrimer $D_{2}[n]$ of the generations $G_{3}$ with 3 growth stages.

Theorem 7 Let $\mathrm{n} \geq 1$, then the $A B C_{4}$ index of polyphenylene dendrimers $D_{2}[n]$ is

$$
\begin{gathered}
A B C_{4}\left(D_{2}[n]\right)=2^{n+2}\left(\sqrt{\frac{13}{14}}+4 \sqrt{\frac{11}{35}}\right. \\
\left.+4 \sqrt{\frac{7}{5}}+2 \sqrt{12}+\frac{1}{3} \sqrt{\frac{15}{2}}+\frac{8 \sqrt{2}}{5}+\frac{17}{9}\right) \\
-2\left(2 \sqrt{\frac{13}{14}}+8 \sqrt{\frac{7}{5}}+8 \sqrt{12}+\frac{2}{3} \sqrt{\frac{15}{2}}+\frac{32 \sqrt{2}}{5}+\frac{34}{9}-\frac{\sqrt{3}}{7}-6 \sqrt{\frac{11}{42}}\right)
\end{gathered}
$$

Proof. In the construction of $D_{2}[n]$ from $D_{2}[n$ $1]$, the graph $\mathrm{D}_{2}[\mathrm{n}]$ have the edges of the form $(4,4),(4,5),(5,5),(5,7),(6,7),(6,8),(7,7)$, $(7,8),(7,9)$ and $(8,9)$. Since

$$
\begin{gathered}
A B C_{4}\left(D_{2}[n]\right)=\sum_{u v \in E(G)} \sqrt{\frac{S_{u}+S_{v}-2}{S_{u} S_{v}}} \\
=\sqrt{\frac{7+7-2}{7 \times 7}} \times 1+\sqrt{\frac{7+6-2}{7 \times 6}} \times 12+\sqrt{\frac{7+8-2}{7 \times 8}}\left(82^{n}-8\right) \\
+\sqrt{\frac{6+8-2}{6 \times 8}}\left(82^{n}-8\right)+\sqrt{\frac{7+5-2}{7 \times 5}}\left[16\left(2^{n}-1\right)+16\right] \\
+\sqrt{\frac{5+4-2}{5 \times 4}}\left(322^{n}-32\right)+\sqrt{\frac{4+4-2}{4 \times 4}}\left(322^{n}-32\right) \\
+\sqrt{\frac{8+9-2}{8 \times 9}}\left(82^{n}-8\right)+\sqrt{\frac{9+9-2}{9 \times 9}}\left(82^{n}-8\right) \\
+\sqrt{\frac{9+7-2}{9 \times 7}}\left(122^{n}-12\right)+\sqrt{\frac{5+5-2}{5 \times 5}}\left[16\left(2^{n}-1\right)-16\right] .
\end{gathered}
$$

After simplification, we get

$$
\begin{gathered}
A B C_{4}\left(D_{2}[n]\right)=2^{n+2}\left(\sqrt{\frac{13}{14}}+4 \sqrt{\frac{11}{35}}+4 \sqrt{\frac{7}{5}}\right. \\
\left.+2 \sqrt{12}+\frac{1}{3} \sqrt{\frac{15}{2}}+\frac{8 \sqrt{2}}{5}+\frac{17}{9}\right)
\end{gathered}
$$




$$
-2\left(2 \sqrt{\frac{13}{14}}+8 \sqrt{\frac{7}{5}}+8 \sqrt{12}+\frac{2}{3} \sqrt{\frac{15}{2}}+\frac{32 \sqrt{2}}{5}+\frac{34}{9}-\frac{\sqrt{3}}{7}-6 \sqrt{\frac{11}{42}}\right)
$$

In the next theorem, the $G A_{5}$ index for $D_{2}[n]$ is computed.

Theorem 8 Let $n \geq 1$, then $G A_{5}$ index of $D_{2}[n]$ is

$$
\begin{gathered}
G A_{5}\left(D_{2}[n]\right)=\left(\begin{array}{l}
\frac{32 \sqrt{14}}{15}+\frac{256 \sqrt{3}}{7}+\frac{\sqrt{35}}{3} \times 8 \\
+\frac{128 \sqrt{5}}{9}+\frac{96 \sqrt{2}}{17}+\frac{9 \sqrt{7}}{2}+56
\end{array}\right) 2^{n} \\
-\left(\frac{32 \sqrt{14}}{15}+\frac{256 \sqrt{3}}{7}+\frac{128 \sqrt{5}}{9}+\frac{96 \sqrt{2}}{17}+\frac{9 \sqrt{7}}{2}-\frac{24 \sqrt{42}}{13}+71\right) .
\end{gathered}
$$

Proof. The formula for $G A_{5}$ index of the graph of polyphenylene dendrimers can be reduced in the following form

$$
\begin{gathered}
G A_{5}\left(D_{2}[n]\right)=\frac{2 \sqrt{7 \times 7}}{7+7} \times 1+\frac{2 \sqrt{7 \times 6}}{7+6} \times 12+\frac{2 \sqrt{7 \times 8}}{7+8}\left(82^{n}-8\right) \\
+\frac{2 \sqrt{6 \times 8}}{6+8}\left(82^{n}-8\right)+\frac{2 \sqrt{7 \times 5}}{7+5} \times 162^{n}+\frac{2 \sqrt{5 \times 4}}{5+4}\left(322^{n}-32\right) \\
\quad+\frac{2 \sqrt{4 \times 4}}{4+4}\left(322^{n}-32\right)+\frac{2 \sqrt{8 \times 9}}{8+9}\left(82^{n}-8\right) \\
+\frac{2 \sqrt{9 \times 9}}{9+9}\left(82^{n}-8\right)+\frac{2 \sqrt{9 \times 7}}{9+7}\left(122^{n}-12\right)+\frac{2 \sqrt{5 \times 5}}{5+5}\left(162^{n}-32\right)
\end{gathered}
$$

After simplification, we get

$$
\begin{gathered}
G A_{5}\left(D_{2}[n]\right)=\left(\begin{array}{l}
\frac{32 \sqrt{14}}{15}+\frac{256 \sqrt{3}}{7}+\frac{\sqrt{35}}{3} \times 8 \\
+\frac{128 \sqrt{5}}{9}+\frac{96 \sqrt{2}}{17}+\frac{9 \sqrt{7}}{2}+56
\end{array}\right) 2^{n} \\
-\left(\frac{32 \sqrt{14}}{15}+\frac{256 \sqrt{3}}{7}+\frac{128 \sqrt{5}}{9}+\frac{96 \sqrt{2}}{17}+\frac{9 \sqrt{7}}{2}-\frac{24 \sqrt{42}}{13}+71\right) .
\end{gathered}
$$

\section{Conclusion}

In this paper, certain degree-based topological indices, namely atomic-bond connectivity index $(A B C)$, geometricarithmetic index $(G A)$, the fourth version of $A B C$ index and the fifthe version of $G A$ index for some infinite classes of nanostar dendrimers were studied for the first time and analytical closed formulas for these networks were determined. These results are very helpful in understanding and predicting the physico-chemical properties for these chemical structures. The study of distance related graph indices for these important chemical graphs are still open to work on.

\section{Acknowledgments}

This research is supported by the grant of Higher Education Commission of Pakistan via Ref. No.20-367/NRPU/R D/HEC/12/831 and by National University of Sciences and Technology, Islamabad, Pakistan and also this research is supported by the Start-up Research Grant 2016 of United Arab Emirates University (UAEU), Al Ain, United Arab Emirates via Grant No. G00002233 and UPAR Grant of UAEU via Grant No. G00002590.

\section{Orcid:}

Muhammad Imran:

https://orcid.org/0000-0002-2827-0462

Syed Ahtsham ul haq Bokhary:

https://orcid.org/0000-0002-8957-0792

Muhammad Kamran Siddiqui:

https://orcid.org/0000-0002-2607-4847

\section{References}

[1] S. Alikhani, R. Hasni, N.E. Arif, J. Comput. Theor. Nanosci., 2014, 11, 1802-1805.

[2] S. A. Bokhary, M. Imran, S. Manzoor, Can. J. Chem., 2016, 94, 120-125,

[3] M. Baca, J. Horváthová, M. Mokrišová, Appl. Math. Comput., 2015, 251, 154-61,

[4] A.Q. Baig, M. Imran, H. Ali, Optoelectron. Adv. Mater. Rapid. Communin., 2015, 9, 248255.

[5] A.Q. Baig, M. Imran, H. Ali, Canad. J. Chem., 2015, 93, 730-739.

[6] B. Bollobás, P. Erdös, Ars. Combin., 1998, 50, 225-33.

[7] G. Caporossi, I. Gutman, P. Hansen, L. Pavlovíc, Comput. Bio. Chem., 2003, 27, 85-90. [8] M. Deza, P.W. Fowler, A. Rassat, K.M. Rogers, J. Chem. Inf. Comput. Sci., 2000, 40, 550-558.

[9] K.C. Das, I. Gutman, B. Furtula, MATCH Commun. Math. Comput. Chem., 2011, 65, 595-644.

[10] M. Ghorbani, A. Khaki, Optoelectron. Adv. Mater. Rapid. Commun., 2010, 4, 2212-2215.

[11] M.V. Diudea, I. Gutman, J. Lorentz, Molecular topology, nova, Huntington. 2001. 
[12] E. Estrada, L. Torres, L. Rodríguez, I. Gutman, Indian. J. Chem., 1998, 37A, 849-855.

[13] B. Furtula, I. Gutman, J. Chemometrics, 2011, 25, 87-91.

[14] M. Ghorbani, M.A. Hosseinzadeh, Optoelectron. Adv. Mater. Rapid. Commun., 2010, 4, 1419-1422.

[15] A. Graovac, M. Ghorbani, J. Math. Nanosci., 2011, 1, 33-42.

[16] S Hayat, M. Imran, J. Comput. Theor. Nanosci., 2015, 12, 70-76.

[17] S Hayat, M. Imran, J Comput Theor Nanosci; 2015, 12, 533-41.

[18] S Hayat, M. Imran, J. Comput. Theor. Nanosci., 2015, 12, 1599-1605,

[19] S Hayat, M. Imran, Appl. Math. Comput., 2014, 240, 213-228,

[20] M. Imran, A.Q. Baig, H. Ali, Canad. J. Chem., 2015, 93, 730-739,

[21] M. Imran, S. Hafib, W. Gao, M.R. Farahani, Chaos, Solitons and Fractals; 2017, 98, 199204,

[22] M. Imran, S. Hayat, K. Shafique, Optoelectron. Adv. Mater. Rapid Commun., 2015, 9, 821-830,

[23] A. Iranmanesh, M. Zeraatkar, Optoelectron. Adv. Mater. Rapid Commun., 2010, 4, 1852-1855.
[24] W. Lin, J. Chen, Q. Chen, T. Gao, X. Lin, B. Cai, MATCH Commun. Math. Comput. Chem., 2014, 72, 699-708.

[25] P.D. Manuel, M.I. Abd-El-Barr, I. Rajasingh, B. Rajan, J. Discr. Algo., 2008, 6, 1119.

[26] J.L. Palacios, MATCH Commun. Math. Comput. Chem., 2014, 72, 709-13

[27] D. Vukičević, B. Furtula, J. Math. Chem., 2009, 46, 1369-1376.

How to cite this article: Muhammad Imran*, Syed Ahtsham Ul Haq Bokhary, Sadia Manzoor, Muhammad Kamran Siddiqui. On molecular topological descriptors of certain families of nanostar dendrimers. Eurasian Chemical Communications, 2020, 2(6), 680-687. Link:

http://www.echemcom.com/article_10509 8.html

Copyright (C) 2020 by SPC (Sami Publishing Company) + is an open access article distributed under the Creative Commons Attribution License, which permits unrestricted use, distribution, and reproduction in any medium, provided the original work is properly cited. 